\title{
化学调控淀粉样蛋白聚集及其在阿尔兹海默症 诊断和治疗中的研究进展
}

高楠, 曲晓刚 ${ }^{*}$

中国科学院长春应用化学研究所稀土资源利用国家重点实验室/化学生物学实验室, 长春 130022

*通讯作者, E-mail: xqu@ciac.ac.cn

收稿日期：2018-04-03; 接受日期：2018-04-27; 网络版发表日期: 2018-07-12

国家自然科学基金(编号: 21431007, 21533008)和中国科学院前沿科学重点研究计划(编号: QYZDJ-SSW-SLH052)资助项目

摘要阿尔兹海默症(AD) 又称老年痴呆症, 由德国医生阿尔兹海默于1906年第一次公开报道. 目前 $\mathrm{AD}$ 已成为 现代社会仅次于心血管病、癌症和脑中风的人类健康第四大杀手，而且目前用于照顾 $\mathrm{AD}$ 患者的费用已高于心血 管病、癌症和脑中风这三种疾病的总和. 因此, 寻找针对 $\mathrm{AD}$ 的有效治疗手段刻不容缓。大量研究表明, 过量 $\beta$-淀 粉样蛋白(AB) 的产生、聚集以及由此导致的一系列氧化损伤在发病过程中起着核心作用。文中综述了本课题组 近年来在 $\mathrm{AD}$ 机理、诊断和治疗等方面取得的部分研究进展, 主要包括 $\mathrm{A} \beta$-金属/蛋白/无机药物试剂作用的机理研 究、靶向抑制剂和多功能治疗剂的设计与生物学效应, 以期为构建更高效的治疗体系探索一条新途径.

关键词阿尔兹海默症, $\beta$-淀粉样蛋白, 聚集, 分子间作用机理, 多功能治疗剂

\section{1 引言}

1906年，德国医生阿罗伊斯·阿尔兹海默(Alios Alzheimer)在临床病理报告中首次描述了阿尔兹海默 症(Alzheimer’s disease, AD)即老年痴呆症的病理特征. $\mathrm{AD}$ 是老年人群中最常见的慢性神经退行性疾病, 其早 期临床表现为记忆障碍. 随着病情的发展, 患者继而出 现语言障碍、情绪紊乱、运动障碍、视空间技能损 害, 甚至人格和行为改变等神经精神症状, 严重影响社 交、职业与生活, 并使身体机能逐渐丧失, 最终导致死 亡．截至 2015 年底，全世界大概有 4700 万人遭受着 $\mathrm{AD}$ 的困扰, 其中我国的患者数量已经突破了一千万, 绝对 数量居于世界首位 ${ }^{[1]}$.
更严峻的是, 随着我国逐步迈入老龄化社会, 老年 人群的数量已经开始显著上升, 截至2014年底, 我国官 方公布的统计数据显示, 我国老年人的数量已经突破 2 亿, 且呈现逐年快速增长的趋势，这在客观上也扩大 了 $\mathrm{AD}$ 的潜在患病人群. 而且 $\mathrm{AD}$ 病程时间长(短至三五 年, 长至二三十年的病例都可见报道), 目前尚无彻底 根治的药物, 同时这些患者中绝大多数是大于 65 岁的 老年人，使得 $\mathrm{AD}$ 患者已经成为社会和家庭的严重负 担. 据世界卫生组织的统计数据表明, 时至今日, $\mathrm{AD}$ 已成为现代社会中仅次于心血管病、癌症和脑中风的 人类健康的第四大杀手. 同时，全球目前用于照顾 $\mathrm{AD}$ 患者的费用已经高于前三种疾病的总和. 因此, 寻找这 个疾病的治疗手段已经刻不容缓.

引用格式: Gao N, Qu X. Recent advances on chemically modulation of amyloid aggregation and its applications in diagnosis and treatment of Alzheimer's disease. Sci Sin Chim, 2018, 48: 941-955, doi: 10.1360/N032018-00081 
截至目前, $\mathrm{AD}$ 的确切发病机制还没有完全研究清 楚, 研究人员为此也提出了多种致病机理假说. 其中淀 粉样蛋白级联假说(amyloid cascade hypothesis)占主流 地位, 因为 $\mathrm{AD}$ 的重要病理特征是患者脑内细胞外的老 年斑沉积(senile plaques, SP), 而SP的主要成分是 $A \beta$, 因此多数研究者认为 $\mathrm{A} \beta$ 的过量表达及其聚集沉积形 成老年斑是 $\mathrm{AD}$ 病理的中心环节, 可以引发其他一系列 病理过程, 而其他病理过程反过来又会加速 $\mathrm{A} \beta$ 的沉积, 形成一种恶性的循环，加重了 $\mathrm{AD}$ 病情的发展. 由此可 知, 抑制 $\mathrm{A} \beta$ 的聚集是预防和治疗 $\mathrm{AD}$ 的重要策略, 具有 很好的预防和治疗的前景.

\section{$2 \mathrm{~A} \boldsymbol{\beta}$ 寡聚体的产生与毒性}

$\mathrm{A} \beta$ 由淀粉样前体蛋白 (amyloid precursor protein, APP)经 $\beta$-分泌酶和 $\gamma$-分泌酶的顺次切割产生, 含有 39 43 个氨基酸残基. APP是一类由695 770个氨基酸 构成的I型跨膜糖蛋白，其中 $\mathrm{A} \beta$ 的结构区域起始于细 胞膜外侧, 终止于跨膜区. APP在几乎所有组织内都广 泛存在, 脑组织内表达量最高. APP的代谢主要有两条 途径(图 1$)^{[2]}$. 在正常生理条件下, 大多数成熟的APP首 先被 $\alpha$-分泌酶切割, 随后残余在细胞膜上的C端APP片 段再被 $\gamma$-分泌酶进一步裂解产生P3. 由于此途径切割 位点在 $\mathrm{A} \beta$ 内, 因此产生的 $\mathrm{P} 3$ 为不完整的 $\mathrm{A} \beta$ 片段, 该途 径也被称为非淀粉源途径. 而另一条切割途径则是 $\mathrm{APP}$ 经由 $\beta$-分泌酶和 $\gamma$-分泌酶共同作用裂解产生A $\beta$. 由于 $\gamma$-分泌酶切割位点不同, 可以产生A $\beta 40$ 或 $A \beta 42 / 43$ 等不同形式, 这一种切割途径也被称为淀粉源途径. 在 某些病理条件下, $\mathrm{A} \beta$ 的产生和清除机制均发生紊乱, 造成其产生和清除失去平衡, 使得 $\mathrm{A} \beta$ 的浓度升高. 而 高浓度的 $\mathrm{A} \beta$ 又会发生聚集，从而产生一系列的细胞毒 性, 进而导致 $\mathrm{AD}$ 的发生和发展.

$\mathrm{A} \beta$ 的神经毒性与其聚集状态直接相关. 在正常生 理状态下, $A \beta$ 单体是没有毒性的. 然而, 当 $A \beta$ 单体形成 具有 $\beta$-折叠结构的聚集体时，其对神经元有明显的毒 性作用，且不同形态和结构的聚集体的毒性不同。近 期研究发现, $\mathrm{A} \beta$ 寡聚体的毒性比成熟纤维的毒性要 大, 是最主要的毒性物种, 能够快速引起神经元死亡. $\mathrm{A} \beta$ 单体和寡聚体还可以诱导DNA的构象转变和DNA 的聚集, 从而产生毒性 ${ }^{[3,4]}$. 既然 $\mathrm{A} \beta$ 的神经毒性与其聚 集状态直接相关，那么抑制 $A \beta$ 的聚集就成为了治疗

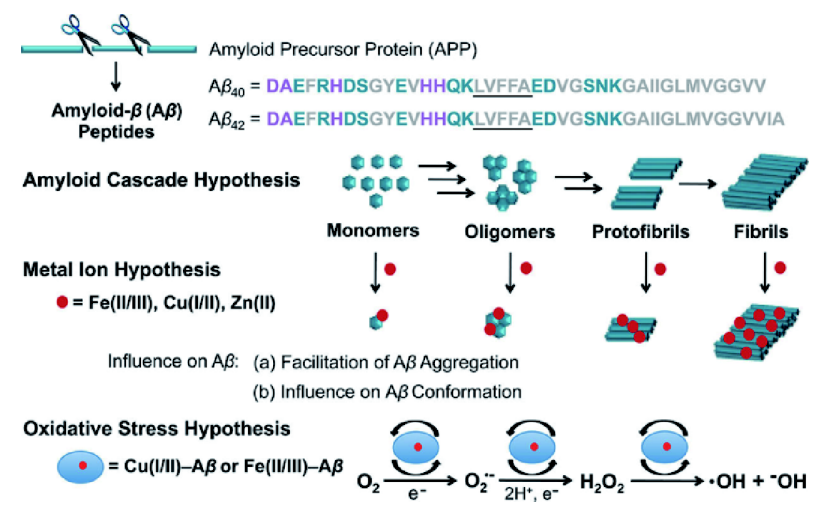

图 1 淀粉样蛋白级联假说、金属离子假说和金属离子- $\mathrm{A} \beta$ 介导的氧化压力假说示意图. $\mathrm{A} \beta 40$ 和 $\mathrm{A} \beta 42$ 的氨基酸序列中, 紫色表示金属结合位点, 蓝色表示亲水氨基酸, 灰色表示输 水氨基酸, 下划线表示自聚集输水区域 ${ }^{[2]}$ (网络版彩图)

Figure 1 Schematic representation of the amyloid cascade hypothesis, the metal ion hypothesis, and the metal-A $\beta$-mediated oxidative stress hypothesis. The amino acid sequences of $A \beta 40 / A \beta 42$ are shown: metal binding residues (purple); hydrophilic and hydrophobic residues (blue and gray, respectively); self-recognition sequence (underlined) [2] (color online)

$\mathrm{AD}$ 的重要手段, 目前也受到最广泛的关注.

因此, 本课题组围绕 $\mathrm{AD}$ 的致病蛋白 $\mathrm{A} \beta$ 进行了一 系列工作，包括基于 $\mathrm{A} \beta$ 的发病机理研究、检测和治疗 等工作.

\section{3 基于 $\mathrm{A} \beta$ 的 $\mathrm{AD}$ 发病机理研究}

由于 $A \beta$ 上具有金属鳌合位点，因此人体内的许多 金属离子如 $\mathrm{Zn}^{2+} 、 \mathrm{Cu}^{2+} 、 \mathrm{Al}^{3+} 、 \mathrm{Fe}^{3+}$ 等都能与 $\mathrm{A} \beta$ 结合 形成 $\mathrm{A} \beta$-金属复合物，在老年斑中也发现了有大量的 金属铜、锌存在，而且金属离子与 $\mathrm{A} \beta$ 的相互作用与 $A \beta$ 毒性的关系也得到了证明. 体外实验发现, 痕量的 金属离子可以促进 $\mathrm{A} \beta$ 聚集, 尤其是 $\mathrm{Zn}^{2+}$, 在很低的浓度 下就可以迅速诱导 $A \beta$ 沉积 ${ }^{[5]}$.

然而，由于 $A \beta$ 自身结构的不确定性和易聚集倾向， 很难得到 $\mathrm{A} \beta$ 与金属结合的晶体结构, 这为确定金属离 子在 $A \beta$ 上的结合位点增加了难度. 之前的研究表明, $\mathrm{A} \beta$ 的 $\mathrm{N}$ 端序列上的 3 个组氨酸残基His6、His 13、 $\mathrm{His} 14$ 是 $\mathrm{Cu}^{2+}$ 与 $\mathrm{Zn}^{2+}$ 的主要结合位点. 除此之外, $\mathrm{N}$ 端的 酪氨酸Tyr 10 和天冬氨酸Asp 1 等也都有可能参与配位. 但同时大量的实验表明，这两种金属与 $\mathrm{A} \beta$ 无论是结合 方式、形成复合物的结构还是在 $\mathrm{AD}$ 中的作用都不是 完全相同的. 
之前的研究表明，金属 $\mathrm{Cu}^{2+}$ 和 $\mathrm{Zn}^{2+}$ 与 $\mathrm{A} \beta$ 结合会引 起 $\mathrm{A} \beta$ 的去质子化 ${ }^{[5]}$. 水分子的物理和化学性质在维持 水溶液中蛋白分子构象、结构动力学以及功能活性等 方面起重要作用, 水合程度会通过影响溶液中的氢 键、静电、疏水和范德华力等弱相互作用来影响蛋白 质的三维结构和稳定性. $\mathrm{A} \beta$ 需要水的参与来维持其结 构，降低溶液中水的活度会促使 $\mathrm{A} \beta$ 从自由状态向 $\beta$-折 叠结构转变, 并促进 $\mathrm{A} \beta$ 纤维化分子间聚集. 因此, 揭示 金属离子与 $\mathrm{A} \beta$ 作用过程中的水合变化能为理解金属 离子在 $\mathrm{AD}$ 中的作用机制提供了参考, 而迄今为止还没 有关于金属离子与 $\mathrm{A} \beta$ 作用过程中直接水合变化的 报道.

本课题组利用渗透压的方法发现了金属锌和铜与 $\mathrm{A} \beta$ 作用都会导致 $\mathrm{A} \beta$ 释放水分子, 并且两种金属结合引 起的水合变化不同. $\mathrm{A} \beta$ 结合金属锌比结合铜引起的失 水数目的两倍还要多. 另外, 通过实验还发现, 金属的 结合会剧烈改变 $A \beta$ 的结构, 使 $A \beta$ 变得不稳定而倾向于 分子间聚集. 而 $A \beta$ 与锌形成的复合物比与铜形成的复 合物更加不稳定、更易于聚集，这与它们在结合过程 中显著的水合变化差异是一致的(图2) ${ }^{[5]}$.

最近的临床研究表明, 胰岛素与AD的发病机理有 关, 而且胰岛素可以保护病人免受 $A \beta$ 诱发的氧化应激. 因此, 我们系统地研究了胰岛素和 $A \beta$ 如何在手性界面 上共同聚集, 用于模拟二者在细胞膜上的纤维化. 实验 结果显示, 表面手性对胰岛素和 $\mathrm{A} \beta$ 共同聚集体的聚集 动力学、二级结构、形态和细胞响应都有很大的影 响. 在 $D$-苯丙氨酸修饰的表面上, $\mathrm{A} \beta$ 会诱导胰岛素聚 集为富含 $\beta$-折叠的纤维, 并且纤维表现出明显的细胞 毒性. 然而, 在 $L$-苯丙氨酸修饰的表面上, 胰岛素和 $\mathrm{A} \beta$

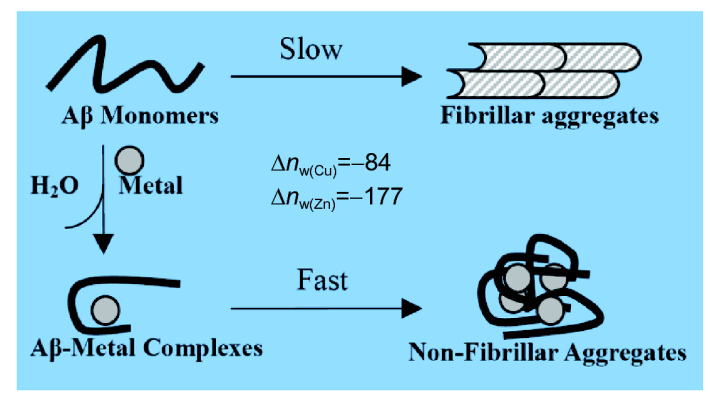

图 $2 \mathrm{~A} \beta$ 在有金属离子或者无金属离子时的聚集途径示意 图 ${ }^{[5]}$ (网络版彩图)

Figure 2 Representative illustration of $A \beta$ aggregation in the absence or presence of metal ions [5] (color online).
则会形成无毒的、无定形的聚集体(图3). 上述结论也 为理解胰岛素与 $\mathrm{AD}$ 之间的关联提供了一种理论上的 解释 ${ }^{[6]}$.

\section{4 基于 $\mathrm{A} \beta$ 的 $\mathrm{AD}$ 诊断研究}

金属离子特别是铜离子和锌离子加速 $\mathrm{A} \beta$ 聚集的 影响已经被广泛研究. 鉴于金属离子在AD患者脑部大 量富集并且可以调节 $\mathrm{A} \beta$ 的构象，快速、低成本、高灵 敏度地检测金属离子诱导的 $\mathrm{A} \beta$ 聚集及其与 $\mathrm{AD}$ 的关系 在临床诊断和治疗中显得十分迫切.

之前的报道显示， $\mathrm{A} \beta$ 中第10位的酪氨酸残基可以 被氧化, 因此可以将其作为检测 $\mathrm{A} \beta$ 的电化学探针. 随 后我们以此为基础, 利用电化学方法研究了金属离子 诱导的 $\mathrm{A} \beta$ 聚集的动力学过程, 并且研究了金属离子诱 导的聚集体与 $\mathrm{A} \beta$ 自身形成的聚集体形貌特征的差异. 结果显示, 当金属离子存在时, $\mathrm{A} \beta$ 聚集的延迟期由于 金属离子快速诱导 $\mathrm{A} \beta$ 聚集而几乎消失. 但是最终的电 流强度却明显高于 $A \beta$ 自身形成的聚集体. 这些结果说 明金属离子可以影响 $\mathrm{A} \beta$ 聚集的动力学和聚集的形貌. 不同的金属离子有不同的效应，并且这种效应可以通 过电化学方法检测出来. 上述研究有助于构建快速、 低成本、高灵敏度的器件, 用于临床诊断金属离子对 阿尔兹海默症及其他与蛋白错误折叠相关的神经退行 性疾病的影响 ${ }^{[7]}$.

当利用传统的四氢噻吩(thioflavin $T, T h T$ )对 $A \beta$ 的

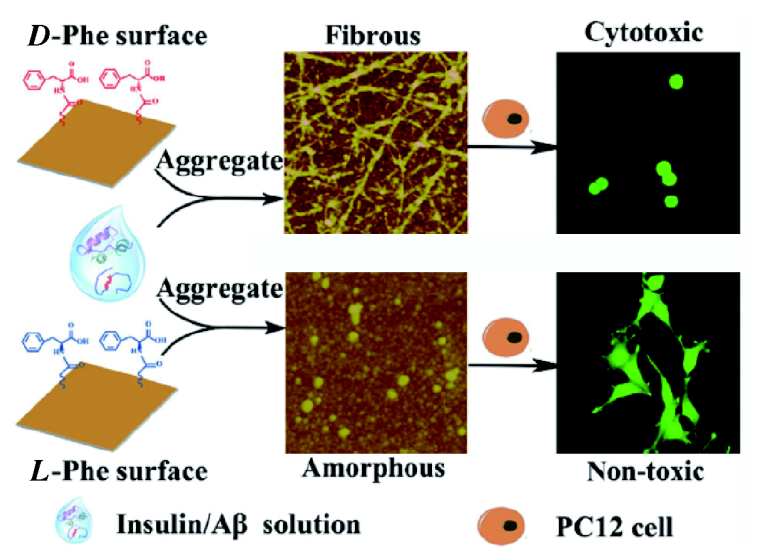

图 3 不同手性界面对于胰岛素- $\mathrm{A} \beta$ 共孵育形态及生物学效 应的影响 ${ }^{[6]}$ (网络版彩图)

Figure 3 The morphology and cellular responses of cross-aggregates of A $\beta$ and insulin on $D$ - and $L$-Phe surfaces [6] (color online). 
聚集程度进行检测时，由于ThT发出的绿色荧光容易 被细胞中其他的内生苂光掩盖，限制了ThT在活体细 胞内的检测效果. 因此，具备红移荧光发射的 $A \beta$ 探针 就将具有很大的优势. 我们在研究中发现, 利用平面结 构的 $N$-甲基卟啉二丙酸 $(N$-methyl mesoporphyrin IX, $\mathrm{NMM}$ ), 可以对细胞中的 $\mathrm{A} \beta$ 进行实时成像与检测(图4). 我们的研究结果为设计和篮选新的监测蛋白质构象紊 乱疾病的试剂提供了新的途径 ${ }^{[8]}$.

此外，利用其他手段，如菲探针通过嵌插入 $\mathrm{A} \beta$ 纤 维而导致荧光淬灭的性质，可以较为灵敏地检测 $\mathrm{A} \beta$ 的 聚集程度和监测聚集过程等 ${ }^{[9 \sim 11}$.

\section{5 基于 $\mathrm{A} \beta$ 的 $\mathrm{AD}$ 治疗体系}

\section{1 治疗药物的精准释放}

尽管大量的 $\mathrm{AD}$ 治疗药物在体外实验中被证实很 有前景，但是由于它们对大脑中的目标分子缺乏特定 的选择性, 无法区分致病分子(蛋白、糖类、金属离子 等)和参与正常生理功能的正常分子. 长期使用这类治 疗剂会引起毒副作用, 因此为了克服上述缺陷, 本课题 组设计了一系列针对 $\mathrm{AD}$ 相关病理因素的药物分子的 精准释放体系.

近年来，使用纳米载体进行药物投递和可控释放 吸引了越来越多的研究兴趣. 这些纳米尺度的载药体 系在降低药物毒性、位点特异性运输、改善药物生物 分布、提高治疗效率等方面展现了明显的优势．更重
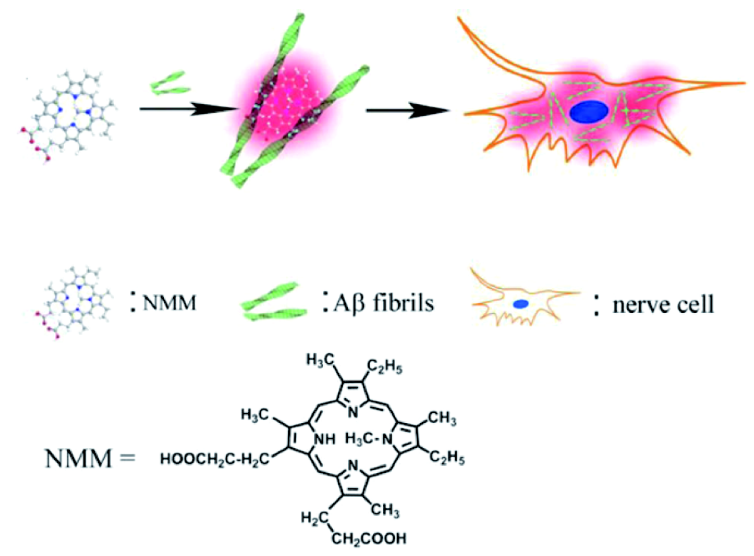

图 4 基于 NMM的在体外和细胞内检测 $\mathrm{A} \beta$ 聚集方法的示意 图 ${ }^{[8]}$ (网络版彩图)

Figure 4 Schematic illustration of NMM-based strategy for sensing A 340 aggregates both in vitro and in cells [8] (color online).
要的是, 由于 $\mathrm{AD}$ 是发生在脑内的疾病, 绝大多数的药 物都会因为无法穿透血脑屏障(blood-brain barrier, BBB)而失去治疗作用. 而纳米载体却被证明具有良好 的穿过BBB的能力，这就有可能克服很多治疗剂的缺 陷, 推动 $\mathrm{AD}$ 治疗的发展.

由于金属离子在 $\mathrm{A} \beta$ 沉积和神经毒性中起到了重 要作用. 之前的研究指出, 在老年斑中 $\mathrm{Cu}^{2+}$ 明显增加且 能够促进 $A \beta$ 的聚集. 而一些金属螯合剂如氯碘喹啉 (clioquinol，CQ)、去铁草酰胺能够显著提高脑组织中 $\mathrm{A} \beta$ 沉淀的再溶解, 并在转基因老鼠和临床试验上取得 了一定的成果. 尽管这些螯合剂很有前途, 但它们对大 脑中的金属离子并没有特定的选择性，无法区分与 $\mathrm{A} \beta$ 斑块关联的有毒金属离子和参与正常生理功能的金属 离子.

为了克服这个缺陷，前期一些科研团队引入了一 种基于螯合剂前体的新理念，螯合剂前体只有特定的 情况，如光照条件下才会激活它的鳌合基团. 药物以 被屏蔽的状态运载到细胞和组织处，在靶标细胞或者 组织中，通过光活性物质的活化激活前体药物使其成 为具有药理活性的分子. 这种光响应释放体系有许多 优势, 包括非侵入性和时空可控的效果. 因此, 这种技 术可以应用到生物体系并且超越传统的药理学给药方 式. 磁性纳米材料作为一种有效的药物运输载体, 可以 毫无困难地将药物运载到靶标细胞或组织处. 基于上 述分析, 我们利用 $\mathrm{Fe}_{3} \mathrm{O}_{4}$ 磁性纳米粒子构建了一个生物 相容性的光响应药物输送平台, 实现了具有 $\mathrm{AD}$ 疗效的 金属螯合剂的靶向输送. 通过光敏感的邻硝基茮基溴 ( $O$-nitrobenzyl bromide, ONB) 的连接基团, 我们将 $\mathrm{CQ}$ 连接在纳米粒子表面, 并将其用于调控金属离子诱导 的 $A \beta$ 聚集以及神经毒性. ONB基团只有在 $365 \mathrm{~nm}$ 的紫 外光照下才会发生光裂解，邻硝基芐基光致异构化成 邻硝基苯甲醛，暴露出螯合基团，实现了金属螯合剂 的可控释放(图5) ${ }^{[12]}$. 我们的研究结果为设计多功能缓 释系统用于 $\mathrm{AD}$ 治疗提供了新思路 ${ }^{[13]}$.

由于 $\mathrm{A} \beta$ 与 $\mathrm{Cu}^{2+}$ 结合之后，还可以产生具有神经毒 性的活性氧簇(reactive oxygen species, ROS), 过多的 ROS能够引发神经细胞调亡, 导致老年斑的形成 ${ }^{[14,15]}$. 在释放鳌合剂的同时还可以将抗氧化的功能增加进 来. 因此, 我们利用可控药物释放体系的优势结合氧化 铈纳米粒子 (CeONP)的抗氧化活性构建了一个新型的 多功能双运载体系. 该体系能够特异性地对双氧水产 


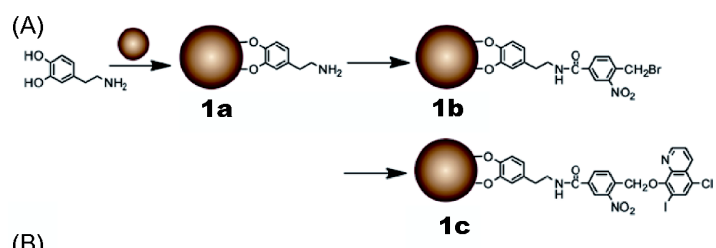

(B)

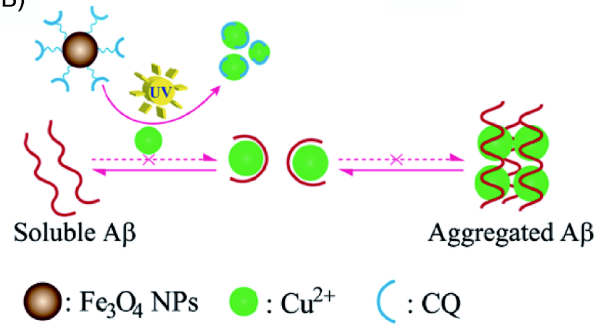

图 5 (a) 表面修饰的磁性纳米粒子体系的合成流程; (b) 基 于磁性纳米粒子的体系可以通过紫外光的调控, 从A $\beta$ 中分 离铜, 进而阻止 $\mathrm{A} \beta$ 聚集 $^{[12]}$ (网络版彩图)

Figure 5 (a) Synthesis of the surface-modified magnetite nanoparticles. (b) The magnetite NPs have low metal affinity, but after UV irradiation, the product sequesters copper from $\mathrm{A} \beta$ so preventing aggregation [12] (color online).

生响应从而释放抗氧化物质和金属螯合剂，实现靶向 治疗 $\mathrm{AD}$. 由于 $\mathrm{AD}$ 发病机理的复杂性，同时针对 $\mathrm{AD}$ 中 的多个靶点, 可以产生协同的治疗作用, 达到最佳疗效 (图6). 研究和开发同时具有作用于 $\mathrm{AD}$ 不同病理环节 的多靶向药物将是抗AD药物发展的新方向. 而在我们 的体系中具有抗聚集性质的金属螯合剂与具有抗氧化 性的氧化铈纳米粒子结合在一个体系中可以达到多靶 向的治疗效果 ${ }^{[16]}$.

大多数的螯合剂既无法穿透血脑屏障，又无法特 异性识别病发区域的有害金属. 因此，我们设计了一 种基于金纳米笼的双响应释放体系，用于可控地释放 金属螯合剂．当体系中存在过氧化氢时，金纳米笼开 始释放螯合剂; 而如果用近红外光照射时，金纳米笼 会产热，从而加速螯合剂的释放。这种智能释放体系 可以有效地抑制 $\mathrm{A} \beta$ 的聚集，减少细胞内的ROS，保护 细胞免受 $A \beta$ 产生的毒性(图7). 鉴于这些优点, 本设计 为远程控制来提高无创治疗 $\mathrm{AD}$ 的疗效提供了新的思 路 ${ }^{[17]}$.

同样的, 我们还设计了一种双功能平台用于 $\mathrm{AD}$ 的 治疗. 利用在石墨烯-介孔硅纳米复合物表面沉积上聚 吡咯, 构建了这个电刺激响应的药物释放平台, 将金属 螯合剂装载进去, 实现了螯合剂的时空可控释放, 利用 螯合剂对金属的配位作用清除AD发病区域过量的金 属离子. 而且电刺激可以诱导神经细胞的轴突进行生

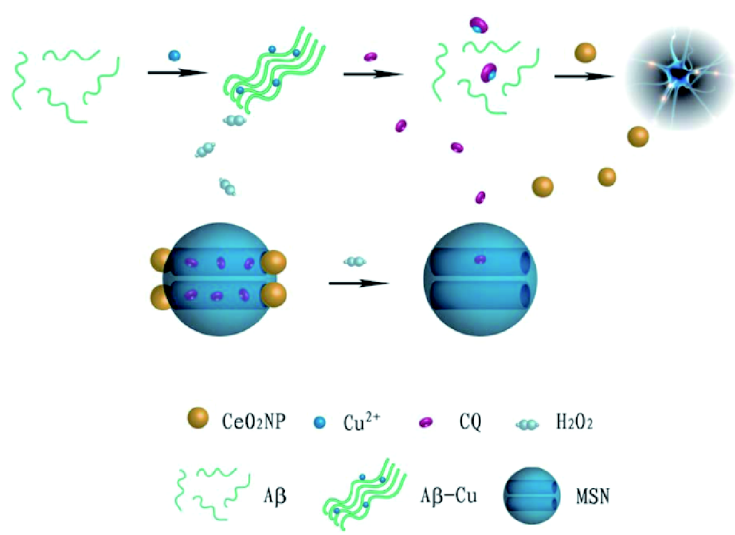

图 $6 \mathrm{H}_{2} \mathrm{O}_{2}$ 响应的基于氧化铈-介孔硅纳米体系的 $C Q$ 智能释 放示意图(网络版彩图)

Figure 6 Schematic representation of $\mathrm{H}_{2} \mathrm{O}_{2}$-fueled release of guest molecules CQ from the pores of mesoporous silica nanoparticles capped with cerium oxide nanoparticles (color online).

(a)

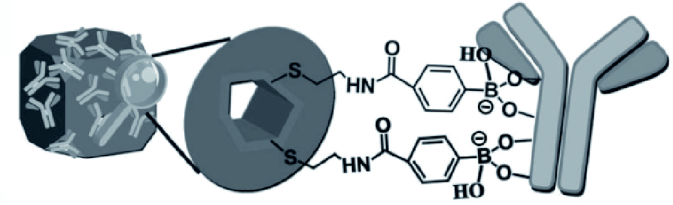

(b)

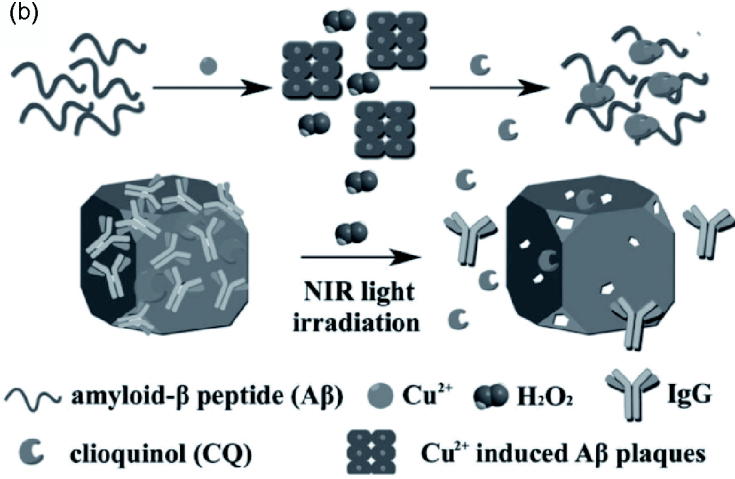

图 $7 \mathrm{H}_{2} \mathrm{O}_{2}$ 和光热双响应的基于金纳米笼的 $\mathrm{CQ}$ 智能释放体 系的构建与工作原理 ${ }^{[17]}$ (网络版彩图)

Figure 7 Schematic representation of $\mathrm{H}_{2} \mathrm{O}_{2}$-fueled and photothermalresponsive release of guest molecules clioquinol from gold nanocageIgG [17] (color online).

长. 该平台可以有效地抑制 $A \beta$ 的聚集，降低细胞内的 ROS水平，保护细胞免受 $A \beta$ 产生毒性的影响 ${ }^{[18]}$. 本研 究促进了 $\mathrm{AD}$ 治疗的无创性遥控多功能系统的设计 (图8).

之前的报道显示，多酚类化合物可以抑制 $\mathrm{A} \beta$ 聚集 和降低ROS，因此近年来也受到了研究人员的广泛重 视. 然而，淀粉样斑块中的过量金属离子(如 $\mathrm{Cu} 、 \mathrm{Zn}$ 


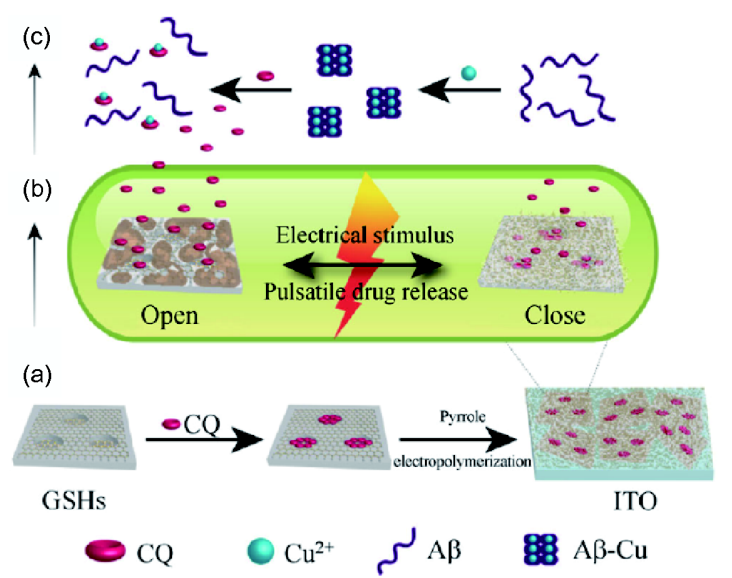

图 8 电脉冲给药系统在AD治疗中的应用 ${ }^{[18]}$ (网络版彩图) Figure 8 Electrically controlled pulsatile drug delivery system for AD therapy [18] (color online).

等)可以与多酚化合物结合，影响多酚化合物的功效. 鉴于此，我们设计和合成了基于介孔硅的、近红外 (near-infrared, NIR)响应的、逐级释放系统UCNP@$\mathrm{SiO}_{2} @ \mathrm{Cur} / \mathrm{CQ}$, 通过调节NIR激光的强度来控制药物 顺序释放. 在该系统中，当用 $0.2 \mathrm{~W}$ NIR光照射时，金 属螯合剂 $\mathrm{CQ}$ 先释放, 用于螯合淀粉样蛋白斑中过量的 金属离子; 随后用 $3 \mathrm{~W}$ NIR光照射, 释放出多酚药物(如 姜黄素). 由于过量的金属离子已经事先被CQ清除, 因 此利用该系统可以增强多酚类药物抑制 $\mathrm{A} \beta$ 聚集和清 除ROS的效果 ${ }^{[19]}$.

\section{2 构建 $\mathrm{A} \beta$ 聚集抑制剂的高效篮选体系}

除了使用传统的治疗剂, 我们还可以根据 $\mathrm{A} \beta$ 的特 点，有针对性地设计新型的 $\mathrm{AD}$ 治疗剂，其中涉及最多 的就是 $A \beta$ 的抑制剂. 但是如何快速鉴定抑制剂的效果 仍然是一个难题. 之前报道的方法在进行 $A \beta$ 抑制剂的 大规模、高通量篎选时或是费时费力，或是需要特殊 的仪器设备. 因此，我们设计了一种简单易行的基于 纳米金的可视化的非标记的高通量 $A \beta$ 抑制剂篮选 体系.

$\mathrm{A} \beta$ 的12 28区域(VHHQKLVFFAEDVGSNK), 是 $\mathrm{A} \beta$ 结构向 $\beta$-片层转化和导致 $\mathrm{A} \beta$ 聚集的重要功能区域. 在 $\mathrm{pH}=5.0$ 时, $\mathrm{A} \beta$ 12 28带正电荷, 其结构主要是由无规 卷曲向 $\beta$-片层转化. 纳米金由于表面被柠檬酸包覆而 带负电荷，而 $\mathrm{A} \beta$ 12 28寡聚体可以看作是聚阳离子, 利用 $\mathrm{A} \beta$ 12 28中与纳米金表面电荷会导致纳米金发
生聚集．在水溶液中，由于入射可见光导致纳米金在 $520 \mathrm{~nm}$ 附近处有着非常强的表面等离子共振，当纳米 金发生聚集时，会观察到吸收峰发生明显红移，从而 导致整个纳米金水溶液的颜色发生改变. 当体系中存 在 $A \beta$ 抑制剂时，可以抑制 $A \beta$ 向 $\beta$-片层结构转化以及塞 聚体的形成, 从而使得纳米金不能发生聚集(图9 ${ }^{[20]}$. 该体系为高通量篮选 $\mathrm{A} \beta$ 抑制剂的工作开辟了一条新 道路.

\section{3 多聚金属氧酸盐类 $\mathrm{A} \beta$ 治疗剂}

自从发现顺铂的抗肿瘤活性以来，许多无机化合 物被用来作为重要的诊断试剂和治疗药物 ${ }^{[21]}$. 在世界 范围内, 无机药物的销售迅速增长. 尽管如此, 只有少 量文献报道了无机药物在抑制 $\mathrm{A} \beta$ 纤维化方面的应用. 作为一种重要的无机化合物, 多聚金属氧酸盐(polyoxometalates，POM)因其在抗病毒、抗肿瘤甚至在抗艾 滋病毒方面都表现出了显著的效果, 从而引起广泛的 关注. 因此，POM可能为我们开启了一条新的、廉价 的治疗多种疾病的道路. 然而, 在当时还没有报道

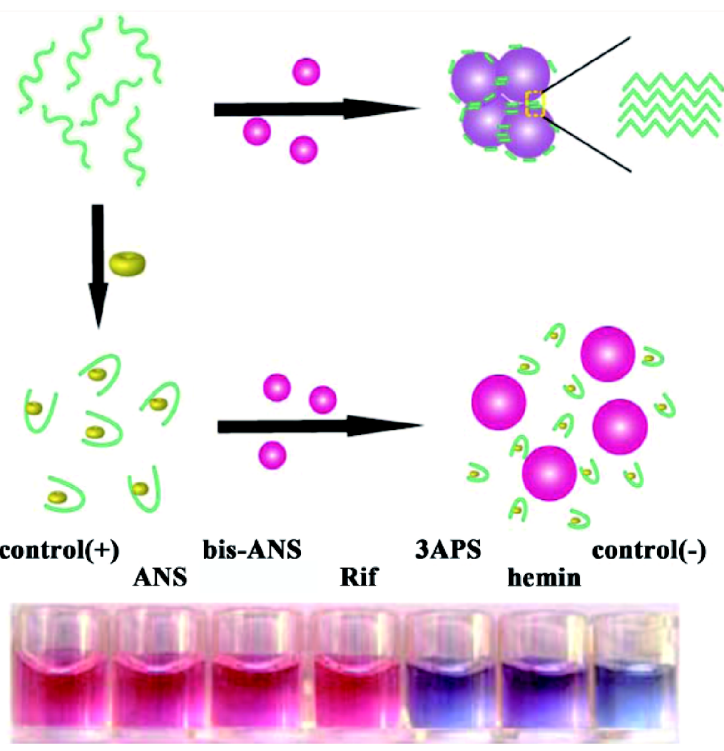

图 9 基于纳米金的 $\mathrm{A} \beta$ 抑制剂快速節选体系(图中红球代表 纳米金, 蓝线代表 $\mathrm{A} \beta$, 黄色圆环代表 $\mathrm{A} \beta$ 抑制剂. 下图为在不 存在或存在配体和 $\mathrm{A} \beta(12 \sim 28)$ 混合溶液的情况下, 纳米金的 色度响应情况 ${ }^{[20]}$ (网络版彩图)

Figure 9 Schematic illustration of the gold nanoparticles (red balls)based strategy for screening $A \beta$ (cyan lines) aggregation inhibitors (yellow circles). The image below shows the colorimetric response of the AuNPs in the absence or presence of ligands and A $\beta(12-28)$ mixed solution, respectively [20] (color online). 
POM在治疗 $\mathrm{AD}$ 方面的应用.

$\mathrm{POM}$ 是一类前过渡金属和氧形成的阴离子簇，具 有无可比拟的结构多样性和独特的物理化学性质. POM主要的优势特征在于它的极性、氧化还原电 位、表面电荷分布、形状和酸度等都是可调控的，而 上述条件是影响 $\mathrm{POM}$ 与生物靶分子识别和反应的重 要因素.

因此，我们首先利用化学、分子生物学、生物化 学、生物物理和现代波谱学等一系列手段, 篮选了一 系列类型的POM, 结果显示一些特殊结构类型的POM 能够抑制 $A \beta$ 的聚集，且抑制效果与POM的结构以及所 带的电荷数有关: 体积越大, 所带电荷数越多, 抑制效 果越好(图10). 同时还发现配合物与 $\mathrm{A} \beta$ 之间的作用主 要是静电相互作用, 作用位点在12 20残基区，尤其是 13 16位的正电荷簇“组氨酸-组氨酸-谷氨酰胺-赖氨酸 $(\mathrm{HHQK})$ ”可能为主要的结合位点 ${ }^{[22]}$. 上述工作为 $\mathrm{POM}$ 直接作为 $A \beta$ 抑制剂奠定了基础.

然而, POM和 $A \beta$ 之间的相互作用主要依赖于静电 结合力, 这种作用力虽然很强, 但是缺乏靶向性. 因此, 我们对POM类抑制剂进行了进一步的改进. 对于POM

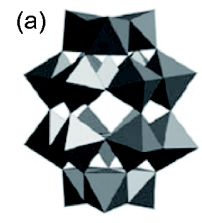

(b)
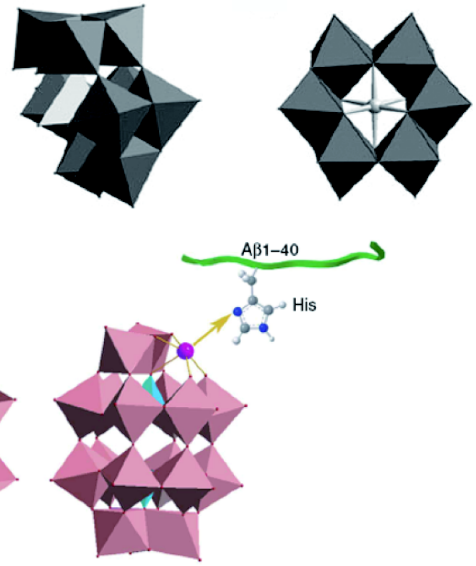

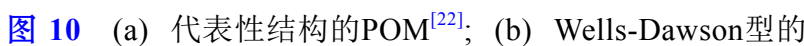
POM的结构，以及通过对Wells-Dawson型POM表面进行过 渡金属修饰(如Ni、Co、 $\mathrm{Cu} 、 \mathrm{Fe}$ 和 $\mathrm{Mn}$ ), 增强对 $\mathrm{A} \beta$ 的识别能 力. 蓝色和亮粉色结构分别代表 $\mathrm{PO}_{4}$ 和 $\mathrm{WO}_{6}$ 的多面体结构; 紫色球体代表组氨酸配位金属; 红色、灰色、黑色和白色 球体分别代表了氧、碳、氮和氢原子(网络版彩图)

Figure 10 (a) Structures of typical polyoxometalates [22]; (b) WellsDawson POM and Wells-Dawson POM with defined histidine-chelating metals (such as $\mathrm{Ni}, \mathrm{Co}, \mathrm{Cu}, \mathrm{Fe}$ and $\mathrm{Mn}$ ). The $\mathrm{PO}_{4}$ and $\mathrm{WO}_{6}$ polyhedra are shown in blue and light pink, respectively. The histidine-chelating metal is shown as a purple ball. The $\mathrm{O}, \mathrm{C}, \mathrm{N}$ and $\mathrm{H}$ atoms are shown as red, grey, dark blue and white balls, respectively (color online).
在 $\mathrm{A} \beta$ 上的作用位点 $\mathrm{HHQK}$ 来说，最大的特点就是存在 着两个连续的组氨酸. 而很多过渡金属原子与组氨酸 之间都存在着很强的螯合作用，因此如果可以在POM 的表面上修饰过渡金属原子，那么这类POM衍生物就 可以增强对 $\mathrm{HHQK}$ 区域的识别能力, 从而增强了对 $\mathrm{A} \beta$ 的识别能力. 实验结果也证实了, 通过修饰不同的过 渡金属原子, POM的衍生物确实可以加强对 $\mathrm{A} \beta$ 的 HHQK区域的识别能力, 尤其是 Co 和 Ni的POM衍生 物. 此外, 我们还利用理论模拟的手段, 第一次得到了 $\mathrm{POM}$ 与 $\mathrm{A} \beta$ 蛋白相互作用的能量最低态模型(图11). 同 时还第一次证明了 $\mathrm{POM}$ 衍生物可以跨过 $\mathrm{BBB}^{[23]}$.

在随后的研究中发现, 单独的POM穿透BBB的能 力毕竟还是有限, 而且仅仅依靠识别HHQK区域也难 以进一步增强抑制剂对于 $A \beta$ 的识别能力. 为了解决上 述问题, 我们随后设计将 $\mathrm{POM}$ 与另一种 $\mathrm{A} \beta$ 靶向肽 LPFFD通过纳米金的载体连接起来，这样既可以解决 靶向识别能力不足的问题，因为LPFFD在 $A \beta$ 上的识别 位点(17 20位)刚好紧邻 $P O M$ 在 $A \beta$ 上的识别位点 (13 16位)，使得新型抑制剂拥有更长的识别区域; 同 时利用纳米金作为载体，有效地增强了抑制剂穿透血 脑屏障的能力, 为传统抑制剂的应用开辟了新的途 径 ${ }^{[24]}$.

随后利用自组装技术开发出了一种更为简单的制 备POM-A $\beta$ 靶向聚集阻断肽复合物的方法. 我们选取 了另一种 $A \beta$ 靶向聚集阻断肽 $Q K L V F F ，$ 利用它 $\mathrm{N}$ 端的 正电荷与带负电的POM进行自组装，形成了新型的纳 米杂化材料(图12). 该材料不但拥有穿越BBB的能力, 还可以增加POM和肽的局部质量密度, 从而提高在小 鼠脑脊液中对 $\mathrm{A} \beta$ 的靶向抑制效果 ${ }^{[25]}$.

\section{4 金属超分子螺旋手性化合物类Aß治疗剂}

然而, 前期的研究普遍忽略了一个问题, 即手性在 $\mathrm{A} \beta$ 聚集中也发挥着十分重要的作用. 手性的作用主要 体现在三个方面：(1) 构成 $\mathrm{A} \beta$ 自身的氨基酸都是手性 的(天然 $\mathrm{L}$ 型氨基酸), 且 $\mathrm{A} \beta$ 形成的二级结构 $(\alpha-$ 螺旋、 $\beta$-折叠等)也是手性的; (2) 由于 $A \beta$ 的手性性质, 使得 $A \beta$ 对手性环境比较敏感, $A \beta$ 的聚集过程自然也会受到 手性的影响; (3) 在近年来的临床研究中发现, 手性对 于药物的疗效也有着十分重要的影响, 手性药物往往 只有一个对映体是有药理效果的, 另一个对映体没有 活性或甚至有毒副作用. 为了减少药物的毒副作用, 

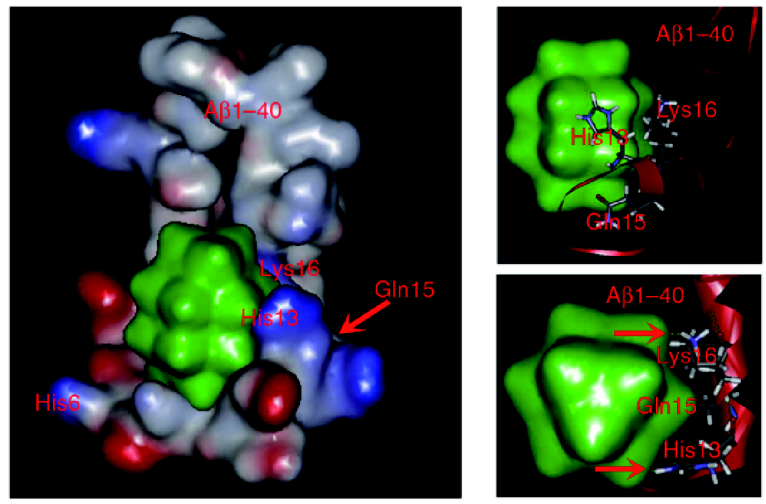

图 $11 \mathrm{~A} \beta$ 与 $\mathrm{POM}$ 相互作用的能量最低态模型. 通过该模型 可以看出POM结合在 $A \beta$ 的一个带正点的空穴部位，而这个 空穴是由 13 位的组氨酸、15位的谷氨酰胺和 16 位的赖氨酸 组成的. 同时POM和 $\mathrm{A} \beta$ 之间还存在着两对氢键, 分别产生于 POM-13 位组氨酸和POM-16位的赖氨酸之间 ${ }^{[23]}$ (网络版 彩图)

Figure 11 Energy-minimized average model of POM-A $\beta$ interactions. A remarkable feature of the model is that the POM-binding site of $A \beta$ is identified as a positive electricity cavity-like domain mainly encompassed by His13, Gln15, Lys16 of A $\beta 1-40$. The complex is further stabilized by two hydrogen bonds, involving His13-POM and Lys16POM [23] (color online).

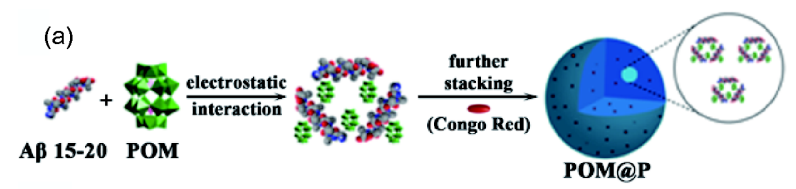

(b)

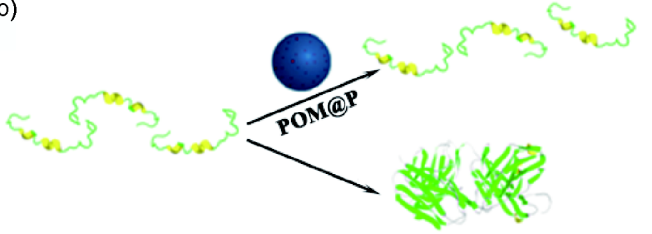

图 $12 \mathrm{~A} \beta$ 靶向聚集阻断肽与POM组装形成纳米粒子并用 于 $A D$ 治疗的示意图 ${ }^{[25]}$ (网络版彩图)

Figure 12 Schematic representation of the peptide-POM conjugates used for $\mathrm{AD}$ treatment [25] (color online).

手性差异性也是关注的重点. 而目前大多数已经报道 的 $\mathrm{A} \beta$ 抑制剂/治疗剂等并没有考虑到手性差异性的 问题.

因此，如何设计一类新型的 $\mathrm{A} \beta$ 抑制剂，使其在兼 顾手性的同时，又能够尽可能多地整合上述已有抑制 剂的优点、避免缺点, 就成为了一项很有挑战性的课 题. 本课题组经过近几年的尝试和摸索，逐渐发现了 一类特定结构的金属超分子螺旋手性化合物可以实现
上述设想.

金属超分子螺旋手性化合物是由Lehn于1987年定 义的. 它是由一个或多个配体链与两个或两个以上金 属离子配位所形成的具有螺旋链状结构的化合物. 金 属超分子螺旋化合物因其所含的金属离子和特异性结 构等具有不一般的光、电和磁性质. 此外, 其特有的螺 旋特性和自组装特性与一些核酸和蛋白等生物分子的 二级结构具有一定的相似性，使其在与复杂结构的核 酸或蛋白分子识别领域有着独特的优势。近年来，金 属超分子螺旋手性化合物在生物医药领域的应用得到 了广泛的重视.

在用于 $\mathrm{AD}$ 治疗剂的开发方面, 金属超分子螺旋手 性化合物具备以下三方面的优势. (1) 金属超分子螺旋 手性化合物可调节的尺寸以及自带的手性性质，可以 实现对 $A \beta$ 手性区域的空间识别与手性识别; (2) 金属 超分子螺旋手性化合物易于进行手性分离，能够得到 纯度较高的单一手性对映体，减少了由手性差异带来 的毒副作用; (3) 金属超分子螺旋手性化合物具备其 他类型抑制剂的优点. 例如，它具备了有机小分子抑 制剂的类药性和水溶性且配体分子具备较好的可修饰 性，可以通过修饰配体来增强对 $\mathrm{A} \beta$ 的靶向性(增加靶 向分子或官能团)、增强对生物屏障的穿透能力(调节 化合物的尺寸与表面性质)、增加多功能性(修饰功能 分子或者官能团)、增加稳定性与分散性(调节表面电 荷和亲疏水性质)和降低生物毒性(手性拆分和螺旋结 构调整). 上述优势也促使我们对金属超分子螺旋手性 化合物在 $\mathrm{AD}$ 中的治疗潜力进行了研究, 并取得了初步 结果.

最近的研究表明, $A \beta$ 的13 23片段属于 $\alpha / \beta$-不一致 区域，这个区域被证明在 $A \beta$ 无聚集倾向时形成 $\alpha$-螺旋 结构，而在 $A \beta$ 发生聚集的前期这段区域可以形成 $\beta$-折 叠结构. 实验证实, 没有13 23位残基的 $\mathrm{A} \beta$ 类似物不形 成淀粉样纤维. 因此 $\alpha / \beta$-不一致区和纤维结构可能存 在着因果关系, 靶向此区域就成为了设计和篮选 $A \beta$ 聚 集抑制剂的新型且有效的途径. 因此, 我们首先报道了 具有锌指结构的两个三螺旋金属超分子手性化合物, $\left[\mathrm{Ni}_{2} \mathrm{~L}_{3}\right]^{4+}$ 和 $\left[\mathrm{Fe}_{2} \mathrm{~L}_{3}\right]^{4+}$ ，能够特异性地结合在 $\mathrm{A} \beta$ 的 $\alpha / \beta$-不 一致伸缩区域，有效地抑制 $\mathrm{A} \beta$ 聚集，解聚已形成的 $\mathrm{A} \beta$ 聚集体(图13)。体内实验结果表明这些化合物可以改 善转基因小鼠模型的空间学习和记忆功能障碍, 并降 低脑内不溶性 $A \beta$ 的含量 ${ }^{[26]}$. 


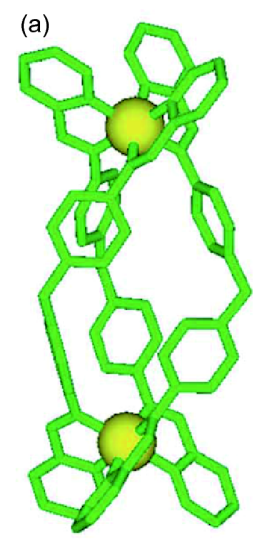

图 $13\left[\mathrm{Ni}_{2} \mathrm{~L}_{3}\right]^{4+}(\mathrm{a})$ 和 $\left[\mathrm{Fe}_{2} \mathrm{~L}_{3}\right]^{4+}(\mathrm{b})$ 的结构示意图 ${ }^{[26]}$ (网络版 彩图)

Figure 13 Structures of $\left[\mathrm{Ni}_{2} \mathrm{~L}_{3}\right]^{4+}$ (a) and $\left[\mathrm{Fe}_{2} \mathrm{~L}_{3}\right]^{4+}$ (b) [26] (color online).

通过上述工作我们发现，如果能够将金属超分子 螺旋手性化合物设计成具有与锌指或 $\alpha$-螺旋等蛋白结 构域相似的结构尺寸, 那么这类金属超分子螺旋化合 物将会更加适合结合在 $A \beta$ 具有 $\alpha$-螺旋结构的 16 23区 域. 同时, $\mathrm{A} \beta \alpha$-螺旋结构的手性和组成肽链的 $L$-氨基 酸所带来的位阻效应可能会导致手性化合物两种对映 体对 $\mathrm{A} \beta$ 产生选择性的识别. 因此, 在接下来的工作中, 通过光学纯的配体与金属自组装, 我们得到了热力学 稳定且手性纯度非常高的具有 $\alpha$-螺旋结构的手性金属 化合物. 随后在之前的工作基础上，利用构建的 $\mathrm{A} \beta$ ECFP高通量细胞篮选抑制剂体系结合生物化学、生 物物理学等研究手段, 发现两对三螺旋金属超分子手 性化合物, $\left[\mathrm{Fe}_{2} \mathrm{~L}_{13}\right]^{4+}$ 和 $\left[\mathrm{Fe}_{2} \mathrm{~L}_{23}\right]^{4+}$ (图14), 能够有效地抑 制 $\mathrm{A} \beta$ 聚集, 并表现出非常明显的手性差异性. 其中 $\Lambda \mathrm{Fe}$ 对映体对 $A \beta$ 聚集的抑制能力强于 $\Delta \mathrm{Fe}$ 对映体. 最后我 们利用实验与理论计算相结合的方式阐明了 $\mathrm{A} \beta$-手性 化合物的作用方式及手性在识别过程中起到的关键作 用 ${ }^{[27 \sim 29]}$.

上面工作中使用的是由刚性配体AB-BA形成的 金属超分子螺旋手性化合物, 然而由于该对称体系的 对称性较高且可进行修饰度较小, 难以实现对结构进 行调节以加强其与 $\mathrm{A} \beta$ 的相互作用以及难以产生供篮 选A $\beta$ 抑制剂的分子库. 最近, 我们开发了一种新的方 法合成光学纯的“头头尾”(head-to-head-to-tail)非对称 结构的金属螺旋超分子化合物. 该化合物的配体是有 方向性的刚性二齿配体AB-CD (图15). 本研究合成并

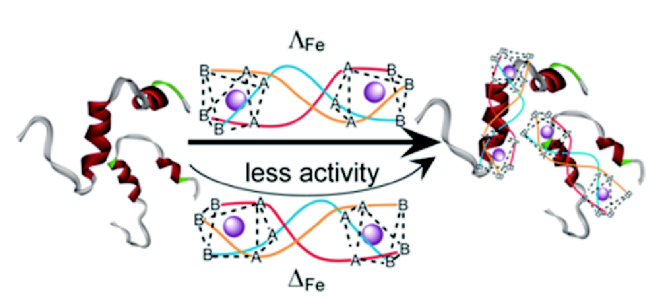

图 14 具有 $\alpha$-螺旋结构的手性金属化合物选择性抑制 $\mathrm{A} \beta$ 聚 集(网络版彩图)

Figure 14 Representative illustration of chiral metallosupramolecular complexes enantioselectively bound to $\mathrm{A} \beta$ (color online).

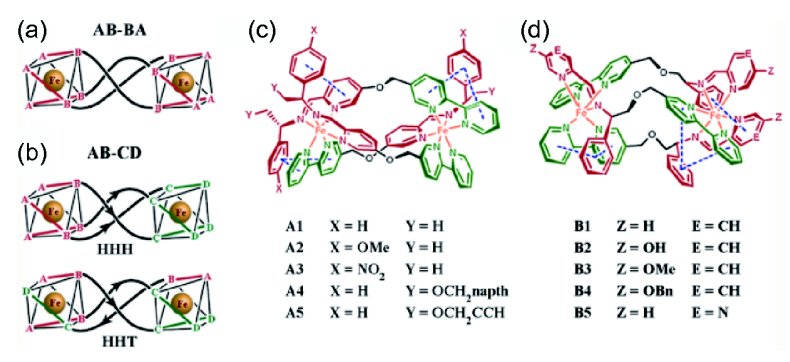

图 15 金属螺旋化合物结构示意图(网络版彩图)

Figure 15 The structures of metallohelices (color online).

表征了10对该类型的非对称金属螺旋对映体，并通过 高通量篮选体系进行了 $\mathrm{A} \beta$ 抑制剂篮选. 实验结果发 现, $\Lambda \mathrm{A} 1$ 和 $\Delta \mathrm{A} 1$ 这对化合物的抑制立体选择性差异最 大，然而 $\Lambda \mathrm{B} 4$ 和 $\Delta \mathrm{B} 4$ 这对化合物的抑制效果是最好的， 体现出了明显的对映体选择性. 随后我们利用实验与 理论计算相结合的方式阐明了选择性的来源, 即 $\pi$ - $\pi$ 堆 积作用在 $A \beta 40$ 中扮演着重要角色, 影响其化学和物理 行为, 而 $\alpha$-螺旋结构以及肽 $L$-氨基酸的手性会导致 $\mathrm{A} \beta 40$ 与 $\Delta / \Lambda$ 对映体之间的疏水作用和 $\pi-\pi$ 相互作用的 不同, 从而产生了对映体选择性的差异(图16). 此外, 这些化合物可以透过血脑屏障, 并阻止 $\mathrm{A} \beta$ 介导的细胞 毒性. 体内研究证明, 这些金属螺旋化合物通过缓解 $\mathrm{A} \beta$ 诱导的毒性延长了CL2006线虫寿命, 也证明了这 类新型不对称手性超分子配合物是一种有潜力的 $\mathrm{AD}$ 治疗剂 ${ }^{[0]}$.

然而, 上述工作仅仅属于初步的尝试, 该领域仍然 存在诸多方面需要进一步改进. 例如, 如何进一步提升 手性化合物对 $A \beta$ 的手性识别能力、赋予手性化合物 的多功能性、增强生物屏障的穿透能力、降低甚至消 除生物毒性、控制手性化合物在生物体内的代谢时间 等, 这些问题都期待着能够在未来的研究中进行探索 和解决. 


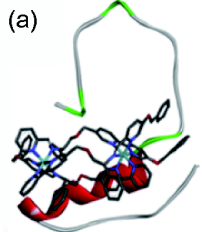

(b)

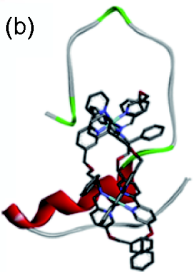

(c)

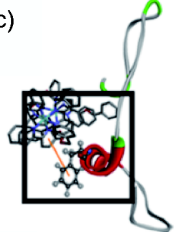

(d)

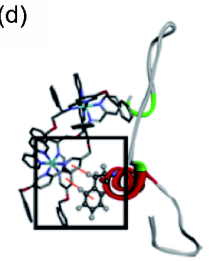

(e)

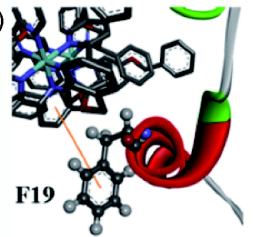

(f)

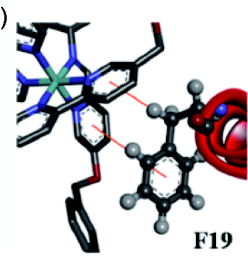

图 16 B4与 $A \beta 40$ 相互作用能量最优化对接模型. (a) A $\beta 40$ 与 $\Lambda \mathrm{B} 4$ 相互作用的能量最低态模型; (b) $\mathrm{A} \beta 40$ 与 $\Delta \mathrm{B} 4$ 相互作 用的能量最低态模型; (c) $\mathrm{A} \beta 40$ 与 $\Lambda \mathrm{B} 4$ 之间的输水相互作用, (e)为(c)中黑框部分的放大视图; (d) $A \beta 40$ 与 $\Delta B 4$ 之间的输水 相互作用, (f)为(d)中黑框部分的放大视图 (网络版彩图)

Figure 16 The interaction of $A \beta 40$ and B4 by docking study. $(a, b)$ Energy minimized average models of (a) $\Lambda \mathrm{B} 4$ and (b) $\triangle \mathrm{B} 4$ with $\mathrm{A} \beta 40$ interactions. (c, d) Hydrophobic interaction between (c) $A \beta 40$ and $\Lambda \mathrm{B} 4$ and between (d) $A \beta 40$ and $\triangle B 4$. (e) Locally amplified image of the black box in (c). (f) Locally amplified image of the black box in (d) (color online).

\section{5 能够水解致病蛋白 $\mathbf{A} \boldsymbol{\beta}$ 的新型治疗剂}

由于抑制剂在 $\mathrm{AD}$ 治疗过程中始终存在着无法克 服的缺点. 例如, 抑制剂改变的只是未聚集与聚集蛋白 之间的比例, 而致病蛋白的总量并没有变化; 同时随着 病情的加重, 致病蛋白的表达量上升, 抑制剂的使用剂 量也需要不断提升, 用以对抗新增的 $\mathrm{A} \beta$ 以及被代谢的 抑制剂，而大剂量的使用药物将会对患者造成额外的 损伤. 因此上述缺陷注定了抑制剂这种治疗方式无法 持久, 这也是抑制剂本质上的缺陷 ${ }^{[31]}$.

因此，我们合成了一种可以水解 $\mathrm{A} \beta$ 单体、寡聚体 和纤维的丝氨酸蛋白酶的纳米模拟酶. 丝氨酸蛋白酶 是蛋白酶家族中种类最多、分布最广的，同时部分天 然 $\mathrm{A} \beta$ 水解酶也属于丝氨酸蛋白酶. 在对丝氨酸蛋白酶 的催化机理进行了研究之后可以发现, 丝氨酸蛋白酶 利用催化三联体Ser-His-Asp水解肽键一共经历了三个 主要的过程: 首先, Ser侧链上带负电的羟基氧亲和进 攻底物肽键中的带正电的羰基碳; 随后，His侧链上带 正电的咪唑基辅助电子进行传递; 最后, Asp带负电的 侧链参与进来辅助稳定过渡态.

根据上述原理，我们合成了单层POM包裹的金纳 米粒子，然后在表面修饰上一种 8 肽分子 $(N$-Ac-CysHis-Sar-His-Sar-His-Sar-His，Sar= $N$-甲基甘氨酸，修饰

时通过 Cys 的-SH与纳米金之间形成的 $\mathrm{S}-\mathrm{Au}$ 键相连), 得到了一种新型的纳米酶(图17) ${ }^{[32]}$.

在这个体系中，纳米酶表面的POM中大量带负电 的氧可以替代Ser进行亲和进攻, 由于POM中的氧的电 负性要高于Ser侧链氧的电负性, 所以POM的亲和进攻 能力会更强(图18); 随后，富含His的八肽中的His可以 起到天然酶催化三联体中His的作用. 由于在生理条件 下POM带负电，而八肽带正电，所以在纳米金表面， $\mathrm{POM}$ 和八肽之间的距离会足够近, 这样也有利于水解 反应时的电子传递; 最后, POM表面大量多余的负电 荷能够替代Asp在水解过程中的作用. 此外, 金纳米粒 子不但可以起到电子传递的作用, 还可以增加整个体 系对于血脑屏障的穿透能力. 因此, 这种纳米酶不但 具有良好的丝氨酸蛋白酶活力, 而且可以水解 $A \beta$ 的单 体和聚集体. 而用来进行修饰的八肽原本就是一种很 好的超氧化物歧化酶(superoxide dismutase, SOD)模拟 肽, 它可以螯合在水解 $\mathrm{A} \beta$ 聚集体过程中产生的过量的 $\mathrm{Cu}$ ，维持体系的离子平衡，并且在八肽螯合了 $\mathrm{Cu}$ 之后， 它会产生明显的SOD活力, 可以快速清除由 $\mathrm{A} \beta$ 产生的 过量的ROS，从而从根本上打破了“A $\mathrm{A} \beta$-ROS恶性循

(a)

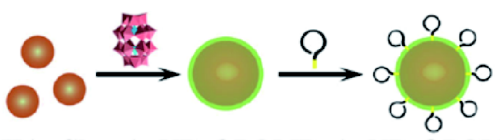

HAuCl 4 AuNPs@POMD AuNPs@POMD-8pep

(b)

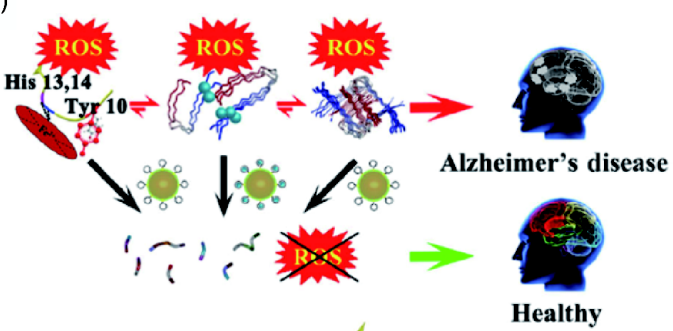

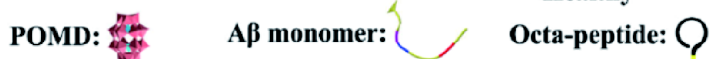

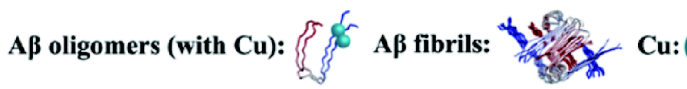

Senile plaques:

As fragments: $(\sim$

Heme:

图 17 AuNPs@POMD-8pep体系的构建及其治疗AD相关 症状的示意图. (a) 纳米酶的合成路线; (b) AuNPs@POMD8 pep作为一个多功能治疗体系, 能够针对 $\mathrm{AD}$ 发病过程中的 多种致病因素 ${ }^{[32]}$ (网络版彩图)

Figure 17 A $\beta$ pathways influenced by AuNPs@POMD-8pep. (a) Synthetic route of the nanozyme. (b) AuNPs@POMD-8pep acted as a multifunctional nanozyme to modulate multiple facets of Alzheimer's disease [32] (color online). 
(a)

(b)
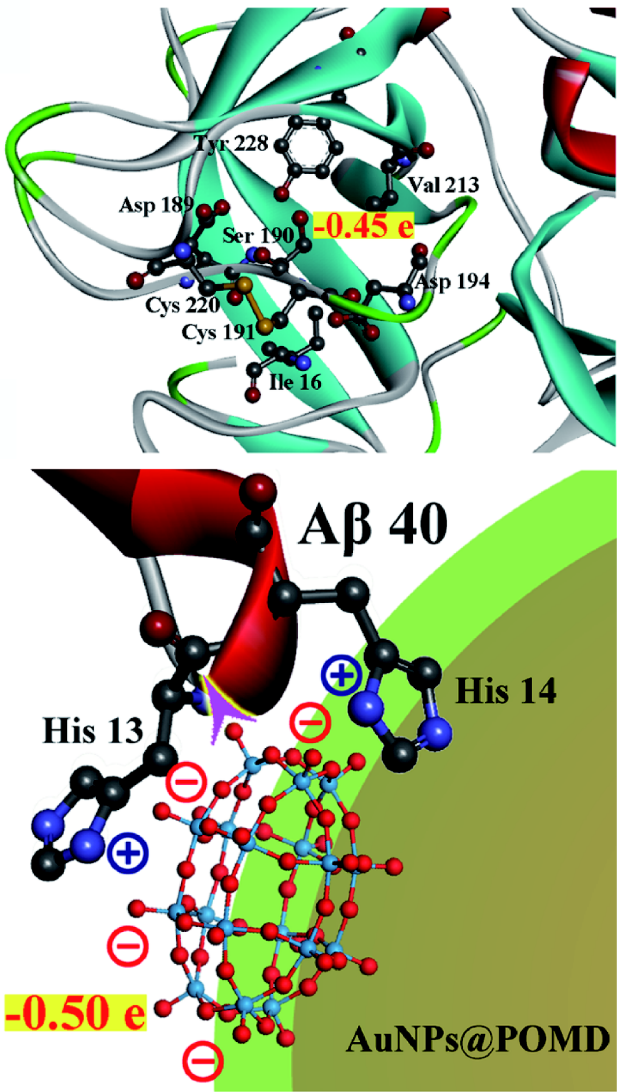

(c)

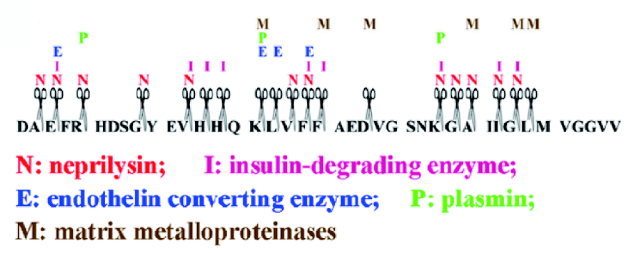

(d)

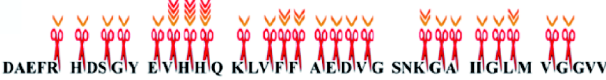

图 18 AuNPs@POMD-8pep体系的工作原理和在A $\beta 40$ 上的 切割位点研究. (a) 天然丝氨酸蛋白酶中催化活性中心 Ser190的电荷分布; (b) POM表面的电荷分布. 图中紫色箭 头表示POM表面带负电的氧原子亲和进攻肽键中带正电的 羰基碳; (c) $\mathrm{A} \beta$ 上存在的主要的天然酶切位点; (d) AuNP$\mathrm{s} @ \mathrm{POMD}-8 \mathrm{pep}$ 在 $\mathrm{A} \beta$ 上的切割位点，切割位点上面标明的箭 头越多，表示在产物分析时该位点出现的时间越早 ${ }^{[32]}$ (网络 版彩图)

Figure 18 Working principle and cleavage sites of AuNPs@POMD8pep on Aß40. (a) Mulliken charge distributions for the Ser190 hydroxyl group in native protease, and (b) the oxygen groups of POM in the nanozyme. The purple arrow in (b) illustrates the electronegative oxygen groups of POM attacking the electropositive carbon atom in the peptide bond. (c) Cleavage sites of the most important A $\beta$-degrading enzymes on A $\beta 40$. (d) Cleavage sites of AuNPs@POMD-8pep on A $\beta 40$. More red arrows are assigned to the sites appearing earlier in the analysis [32] (color online).
环”, 这也为 $\mathrm{AD}$ 的治疗提供了新的思路.

另外根据文献报道, 由于 $\mathrm{Ce}^{3+}$ 和 $\mathrm{Ce}^{4+}$ 离子之间存在 着协同作用, 使得氧化铈纳米粒子(cerium oxide nanoparticles, CeONP)具有抗氧化活性, 可以用来保护大脑 抵抗 $\mathrm{AD}$ 发病过程中的氧化应激. 而这种不同价态的离 子之间的转换也可以用来模拟天然酶中的电子传递过 程. 因此，我们将POM负载到CeONP表面(CeONP@$\mathrm{POMs}$ ), 得到了另一种可以水解 $\mathrm{A} \beta$ 的纳米水解酶(图19). 此外, 由于CeONP还具有抗氧化活性, CeONP@POMs 可以作为一个双功能试剂用于治疗 AD. 体内实验表 明，我们设计的纳米酶具有良好的生物相容性和穿过 $\mathrm{BBB}$ 的能力. 通过对其生物分布、体内毒理学以及模 型动物体重变化的详细研究, 证明了 $\mathrm{CeONP} @ \mathrm{POMs}$ 可作为一种潜在的治疗 $\mathrm{AD}$ 的药物 ${ }^{[33]}$.

\section{6 其他类型的AD治疗剂}

在 $\mathrm{AD}$ 的发病过程中, 除了 $\mathrm{A} \beta$ 表达失衡以外，同样 存在着金属离子的失衡, 在 $\mathrm{AD}$ 病人脑部的 $\mathrm{A} \beta$ 斑块中 就发现了高水平的金属离子(如 $\mathrm{Cu}$ 约 $0.4 \mathrm{mM}$ ). 过量的 金属离子能够与 $\mathrm{A} \beta$ 结合并促进其聚集，并产生高水平 的ROS, 从而损伤蛋白质、DNA和脂质.

根据前文提及的 CeONP的抗氧化活性，我们再次 选择 $\mathrm{CeONP}$ 作为体系的骨架. 而对于金属离子的稳态 失衡问题，传统的处理方法是使用金属螯合剂，然而 由于一般的金属螯合剂的选择性较差，长期使用会带 来很多副作用. 因此, 我们设计了功能化的 $\mathrm{MnMoS}_{4}$ 外 壳，通过离子交换高选择性地移除 $\mathrm{Cu}^{2+}$ ，释放 $\mathrm{Mn}^{2+}$. $\mathrm{Mn}^{2+}$ 能够与细胞表面整联蛋白受体和基膜蛋白相互作 用，因此通过离子交换释放的 $\mathrm{Mn}^{2+}$ 能够促进神经元的 分化，从而保护神经元免受神经突营养障碍和神经元 死亡. 所以，我们首次构建了用 $\mathrm{MnMoS}_{4}$ 功能化的 CeONP纳米材料(CeONPs $\left.@ \mathrm{MnMoS}_{4-n} ; n=1 \sim 5\right)$ 来针对 $\mathrm{AD}$ 的多个因素进行治疗. 该体系能够维持金属离子的 内稳态, 减少氧化应激, 促进细胞分化. CeONP@Mn$\mathrm{MoS}_{4-n}$ 核壳纳米粒子能够通过离子交换高选择性地清 除有毒的 $\mathrm{Cu}^{2+}$ 同时释放有利于增强神经突生长的 $\mathrm{Mn}^{2+}$. 并且, CeONP@MnMoS $\mathrm{Mn}_{4-n}$ 的核心具有SOD活性, 能够 保护细胞免受氧化应激的影响(图20). 与单独的 $\mathrm{MnMoS}_{4}$ 或CeONP比较, 本体系具有协同效果. 上述结 果同样提供了设计多功能系统用于 $\mathrm{AD}$ 治疗的新思 路 ${ }^{[34]}$. 


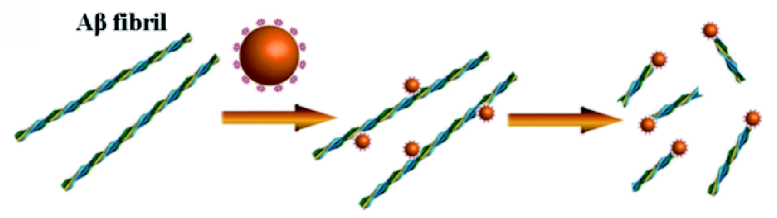

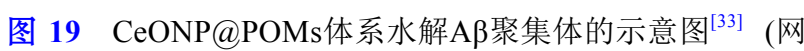
络版彩图)

Figure 19 Scheme of CeONP@POMD as a hydrolytic enzyme to degrade A $\beta 40$ fibrils [33] (color online).
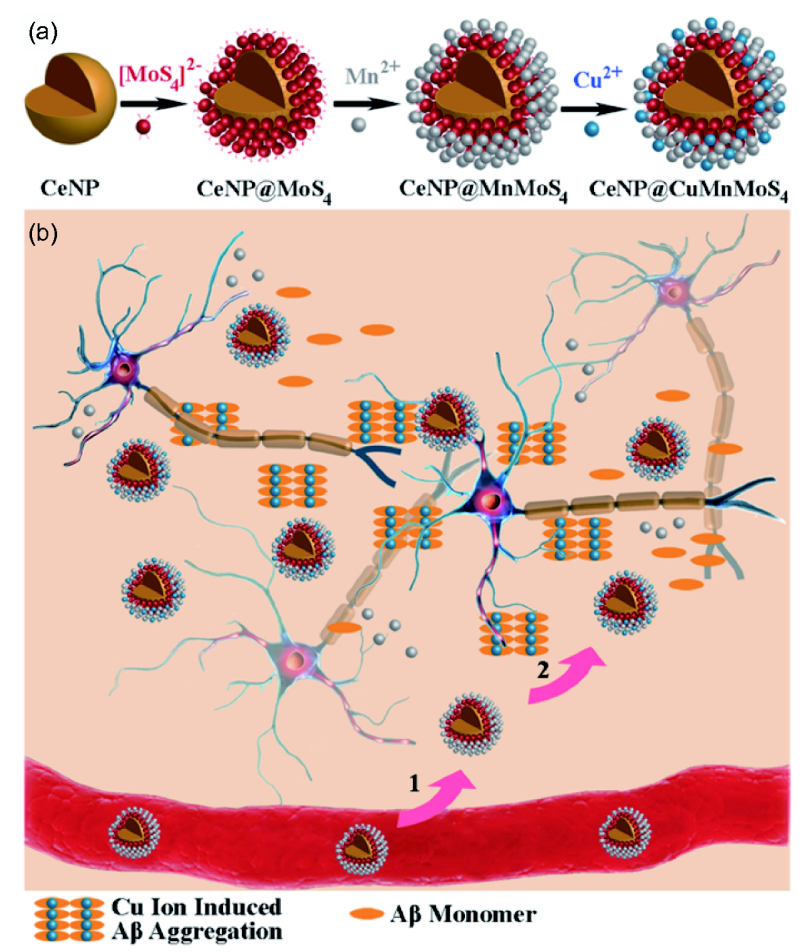

图 20 (a) CeNP@ $\mathrm{MnMoS}_{4-n}$ 的制备. $\mathrm{MnMoS}_{4}$ 的壳层用于清 除 $\mathrm{Cu}^{2+}$ 并释放能够促进细胞分化的 $\mathrm{Mn}^{2+}, \mathrm{CeNP}$ 核心具有 SOD酶活力, 能够清除 $\mathrm{A} \beta-\mathrm{Cu}^{2+}$ 产生的ROS. (b) $\mathrm{CeN}-$ $\mathrm{P} @ \mathrm{Mn} \mathrm{MoS}_{4-n}$ 在体内行使功能的示意图. (1) CeN$\mathrm{P} @ \mathrm{MnMoS}_{4-n}$ 到达疾病区域; (2) $\mathrm{CeNP} @ \mathrm{MnMoS}_{4-n}$ 通过清除 $\mathrm{Cu}^{2+}$ 、释放 $\mathrm{Mn}^{2+}$ 以及清除ROS降低 $\mathrm{A} \beta-\mathrm{Cu}^{2+}$ 的毒性 ${ }^{[34]}$ (网络 版彩图)

Figure 20 (a) The preparation of $\mathrm{CeNP} @ \mathrm{MnMoS}_{4-n}$. $\mathrm{MnMoS}_{4}$ shell was used to remove $\mathrm{Cu}^{2+}$ and release $\mathrm{Mn}^{2+}$ which has the ability to enhance neurite outgrowth. CeNP core perform SOD activity, which can clean the ROS generated by $\mathrm{A} \beta-\mathrm{Cu}^{2+}$. (b) Schematic interpretation of $\mathrm{CeNP} @ \mathrm{MnMoS}_{4-n}$ in vivo: (1) $\mathrm{CeNP} @ \mathrm{MnMoS}_{4-n}$ reach the diseased areas. (2) $\mathrm{CeNP} @ \mathrm{MnMoS}_{4-n}$ decreasing toxicity of $\mathrm{A} \beta-\mathrm{Cu}^{2+}$ by removing $\mathrm{Cu}^{2+}$, releasing $\mathrm{Mn}^{2+}$ and cleaning ROS [34] (color online).

热疗作为当前一种非侵入式治疗癌症的手段, 它 是将生物组织暴露于高于正常温度下来促进选择性地 破坏异常细胞 ${ }^{[35]}$. 近年来, 各种各样的技术如辐射、超
声以及微波等，已经发展成为能升高靶向组织温度的 手段. 虽然这些技术理论上能渗透深层组织，但是需 要很高的频率. 然而由于其扩散性质，这些手段可能 会对周围组织产生有害的高温影响. 为了避免对周围 组织的非特异性的高温影响, 人们探索着采用近红外 频率的激光来照射产热. 近红外光能以足够的强度渗 透组织, 同时对引起局部高温有着更高的空间精确度.

石墨烯作为一种新型的碳纳米材料, 由于其在近 红外区有着较高的光学吸收和光热效率, 使其在生物 医学领域的应用成为研究的重点 ${ }^{[34]}$. 之前的工作已经 开始利用石墨烯强的近红外吸收的性质用于减小小鼠 的肿瘤尺寸和光热消融癌细胞. 然而, 在当时还没有关 于石墨烯的材料用于光热治疗 $\mathrm{AD}$ 的报道. 因此，我们 利用硫黄素S (thioflavin S, ThS) 修饰的氧化石墨烯 (graphene oxide, GO)在近红外光照射下产热来解聚 $\mathrm{A} \beta$ 纤维. $\mathrm{GO}$ 与 $\mathrm{ThS}$ 共价相连形成的复合物能够选择性 地靶向结合 $\mathrm{A} \beta$ 聚集体形成GO-ThS-A $\beta$ 结合物. 随后利 用 $\mathrm{GO}$ 的强的近红外光吸收的性质, 在近红外激光器的 照射下局部产热来解离 $\mathrm{A} \beta$ 纤维(图21). 与传统的化学 治疗和辐射治疗相比, 热疗的优势在于只有受光照的 病变部位才会被治疗, 而其他的组织不受影响, 因此, 它能极大地降低副作用并提高选择性 ${ }^{[36,37]}$.

我们在随后的研究中发现，近红外激光虽然可以 穿透生物组织 ${ }^{[38]}$, 但是对于较深层的组织器官的穿透 能力却较弱. 相比而言, 磁场对于生物组织有着较强 的穿透能力, 因此在AD治疗中, 磁热相比于光热就有 着更明显的优势. 同时，在致病蛋白 $\mathrm{A} \beta$ 的聚集过程中， 寡聚体是目前公认的最具有毒性的阶段，因此特异性 地识别与清除寡聚体被认为是最有效的治疗方法之

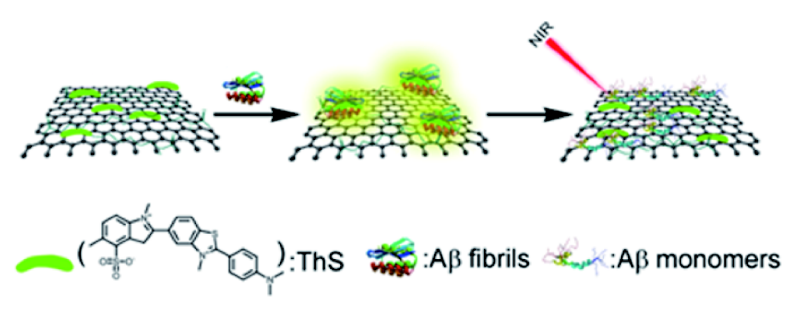

图 21 利用 $\mathrm{ThS}$ 修饰的 $\mathrm{GO}$ 高效的近红外吸收性质光热治疗 $\mathrm{AD}$ 的示意图. GO-ThS靶向结合 $\mathrm{A} \beta$ 聚集体, 并能在近红外光 照下解聚聚集体 ${ }^{[36]}$ (网络版彩图)

Figure 21 Schematic representation of Thioflavin-S modified graphene oxide with high NIR absorbance used for $\mathrm{AD}$ treatment. Graphene oxide-Thioflavin-S can effectively dissolve amyloid deposits of $A \beta 1-40$ upon NIR laser irradiation [36] (color online). 
一. 但是目前对于寡聚体的检测和清除方面的工作所 报道的很少. 因此我们首先设计并合成了一种可以对 $\mathrm{A} \beta$ 寡聚体起特异性苂光响应的小分子化合物NFP, 并 将这个小分子与铁磁纳米粒子相连, 同时实现 $\mathrm{A} \beta$ 寡聚 体的检测与清除. 结果显示, 我们所构建的小分子化合 物NFP对于 $A \beta$ 寡聚体有着特异性的识别能力, 通过对 $A \beta$ 寡聚体和NFP分子进行的分子对接研究结果显示, NFP和A $\beta 19$ 位的苯丙氨酸 $(F 19)$ 之间存在着很强的疏 水相互作用, 这就使得NFP可以嵌插进A $\beta$ 寡聚体中间, 从而实现对寡聚体的特异性识别(图22) ${ }^{[39]}$.

为了进一步增强NFP对于 $A \beta$ 寡聚体的检测能力 以及增加对检测到的寡聚体的解聚能力, 我们将NFP 和 $\mathrm{A} \beta$ 靶向肽 $(K L V F F)$ 连接到了铁磁纳米粒子表面，构 建了新型的治疗体系(MNP@NFP-pep). 该体系一方面 利用纳米粒子优秀的比表面积，增强了NFP的识别能 力; 另一方面利用铁磁纳米粒子优秀的、非介入性的 磁热性质来解聚臭聚体。实验结果显示, 在没有磁场 时，该体系能够高效地识别 $\mathrm{A} \beta$ 寡聚体; 而在外加磁场 时, 又可以将 $\mathrm{A} \beta$ 寡聚体快速降解，从而实现了 $\mathrm{AD}$ 的快 速诊断和治疗的一体化 ${ }^{[39]}$.

\section{6 展望}

随着科技的发展、医疗设施的日益完善、人类寿 命逐渐变长, 人口老龄化的问题也日益突出. 而 $\mathrm{AD}$ 正 是发生于老年期及老年前期的一种进行性中枢神经退 行性疾病, 是老年性痴呆最为常见的形式之一, 且其患 病率随年龄的增加而增加. 随着老龄化社会的来临, $\mathrm{AD}$ 已成为医学上关注的热点问题. 由于 $\mathrm{AD}$ 的病理过 程极其复杂，分子机制尚不明确，对该疾病的治疗受 (a)

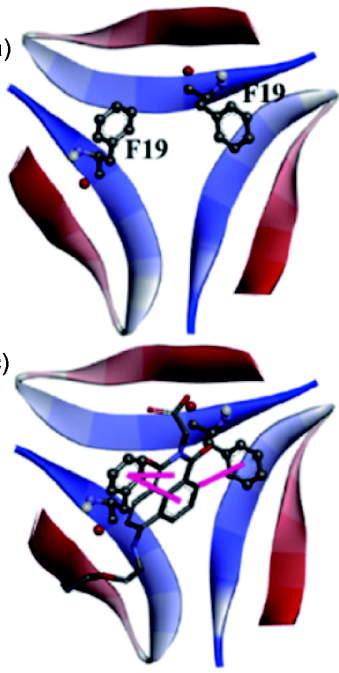

图 22 NFP-A $\beta$ 葟聚体相互作用的能量最低态模型. (a) $A \beta$ 寡聚体晶体结构(PDB ID:4NTR), 蓝色代表 $A \beta$ 单体的N端, 红 色代表 $A \beta$ 单体的C端; (b) NFP小分子结构; (c) NFP-A 3 寡聚 体相互作用的能量最低态示意图(动画模式); (d) NFP-A $\beta$ 葟 聚体相互作用的能量最低态示意图(表面表征模式) ${ }^{[39]}$ (网络 版彩图)

Figure 22 Energy-minimized average model of the NFP-A $\beta$ oligomer interactions. (a) A $\beta$ oligomer observed using X-rays (4NTR). The structure of the N-terminal to the C-terminal is colored in blue to red. (b) Optimized NFP structure. (c) Cartoon and (d) surface representations of the interactions in the complex of NFP and the A $\beta$ oligomers [39] (color online).

到了一定的限制. 但是最近一些成果显示 $A \beta$ 的聚集和 沉积是 $\mathrm{AD}$ 的一个关键性病理事件. 许多因素如 $\mathrm{pH}$ 、 温度、蛋白浓度和金属离子等都可以影响 $\mathrm{A} \beta$ 的聚集. 而其他一些病理特征, 诸如金属浓度紊乱、ROS积累 等都基于 $A \beta$ 的过量表达和聚集的基础之上. 因此，在 未来药物开发的过程中, 以 $\mathrm{A} \beta$ 为基本着眼点，兼顾多 功能的治疗体系有望成为研究的重点.

\section{参考文献}

1 Jia J, Wei C, Chen S, Li F, Tang Y, Qin W, Zhao L, Jin H, Xu H, Wang F, Zhou A, Zuo X, Wu L, Han Y, Han Y, Huang L, Wang Q, Li D, Chu C, Shi L, Gong M, Du Y, Zhang J, Zhang J, Zhou C, Lv J, Lv Y, Xie H, Ji Y, Li F, Yu E, Luo B, Wang Y, Yang S, Qu Q, Guo Q, Liang F, Zhang J, Tan L, Shen L, Zhang K, Zhang J, Peng D, Tang M, Lv P, Fang B, Chu L, Jia L, Gauthier S. Alzheimer's Dementia, 2018, 14: 483-491

2 Savelieff MG, DeToma AS, Derrick JS, Lim MH. Acc Chem Res, 2014, 47: 2475-2482

3 Geng J, Zhao C, Ren J, Qu X. Chem Commun, 2010, 46: 7187-7189

4 Yu H, Ren J, Qu X. Biophys J, 2007, 92: 185-191

5 Yu H, Ren J, Qu X. ChemBioChem, 2008, 9: 879-882

6 Du Z, Guan Y, Ding C, Gao N, Ren J, Qu X. Nano Res, 2018, doi: 10.1007/s12274-018-1995-y

7 Geng J, Yu H, Ren J, Qu X. Electrochem Commun, 2008, 10: 1797-1800

8 Li M, Zhao A, Ren J, Qu X. ACS Chem Neurosci, 2017, 8: 1299-1304 
9 Li M, Zhao C, Yang X, Ren J, Xu C, Qu X. Small, 2013, 9: 52-55

10 Song Y, Qu K, Xu C, Ren J, Qu X. Chem Commun, 2010, 46: 6572-6574

11 Li M, Guan Y, Zhao A, Ren J, Qu X. Theranostics, 2017, 7: 2996-3006

12 Li M, Liu Z, Ren J, Qu X. Chem Sci, 2012, 3: 868-873

13 Xu C, Shi P, Li M, Ren J, Qu X. Nano Res, 2015, 8: 2431-2444

14 Geng J, Li M, Wu L, Chen C, Qu X. Adv Healthc Mater, 2012, 1: 332-336

15 Geng J, Li M, Wu L, Ren J, Qu X. J Med Chem, 2012, 55: 9146-9155

16 Li M, Shi P, Xu C, Ren J, Qu X. Chem Sci, 2013, 4: 2536-2542

17 Shi P, Li M, Ren J, Qu X. Adv Funct Mater, 2013, 23: 5412-5419

18 Wu L, Wang J, Gao N, Ren J, Zhao A, Qu X. Nano Res, 2015, 8: 2400-2414

19 Ma M, Gao N, Sun Y, Ren J, Qu X. Small, 2017, 13: 1701817

20 Geng J, Qu K, Ren J, Qu X. Mol Biosyst, 2010, 6: 2389-2391

21 Li M, Guan Y, Chen Z, Gao N, Ren J, Dong K, Qu X. Nano Res, 2016, 9: 2411-2423

22 Geng J, Li M, Ren J, Wang E, Qu X. Angew Chem Int Ed, 2011, 50: 4184-4188

Gao N, Sun H, Dong K, Ren J, Duan T, Xu C, Qu X. Nat Commun, 2014, 5: 3422

Gao N, Sun H, Dong K, Ren J, Qu X. Chem Eur J, 2015, 21: 829-835

Li M, Xu C, Wu L, Ren J, Wang E, Qu X. Small, 2013, 9: 3455-3461

Yu H, Li M, Liu G, Geng J, Wang J, Ren J, Zhao C, Qu X. Chem Sci, 2012, 3: 3145-3153

Li M, Howson SE, Dong K, Gao N, Ren J, Scott P, Qu X. J Am Chem Soc, 2014, 136: 11655-11663

Li M, Zhao C, Duan T, Ren J, Qu X. Adv Healthc Mater, 2014, 3: 832-836

Li M, Zhao C, Ren J, Qu X. Small, 2015, 11: 4651-4655

Guan Y, Du Z, Gao N, Cao Y, Wang X, Scott P, Song H, Ren J, Qu X. Sci Adv, 2018, 4: eaao6718

Li M, Xu C, Ren J, Wang E, Qu X. Chem Commun, 2013, 49: 11394-11396

Gao N, Dong K, Zhao A, Sun H, Wang Y, Ren J, Qu X. Nano Res, 2016, 9: 1079-1090

Guan Y, Li M, Dong K, Gao N, Ren J, Zheng Y, Qu X. Biomaterials, 2016, 98: 92-102

Guan Y, Gao N, Ren J, Qu X. Chem Eur J, 2016, 22: 14523-14526

Li M, Zhao A, Dong K, Li W, Ren J, Qu X. Nano Res, 2015, 8: 3216-3227

Li M, Yang X, Ren J, Qu K, Qu X. Adv Mater, 2012, 24: 1722-1728

Li M, Guan Y, Ding C, Chen Z, Ren J, Qu X. J Mater Chem B, 2016, 4: 4072-4075

Xu C, Bing W, Wang F, Ren J, Qu X. ACS Nano, 2017, 11: 7770-7780

Du Z, Gao N, Guan Y, Ding C, Sun Y, Ren J, Qu X. Nano Res, 2018, 11: 1987-1997 


\title{
Recent advances on chemically modulation of amyloid aggregation and its applications in diagnosis and treatment of Alzheimer's disease
}

\author{
Nan Gao, Xiaogang $\mathrm{Qu}^{*}$ \\ State Key Laboratory of Rare Earth Resource Utilization/Laboratory of Chemical Biology, Changchun Institute of Applied Chemistry, Chinese \\ Academy of Sciences, Changchun 130022, China \\ *Corresponding author (email: xqu@ciac.ac.cn)
}

\begin{abstract}
Alzheimer's disease (AD) is characterized by cerebral extracellular amyloid plaques and intracellular neurofibrillary tangles. Although the molecular mechanisms of AD pathogenesis are not clearly understood owing to its complexity, recent studies have demonstrated that the polymerization of amyloid $\beta$-peptides $(A \beta)$ into amyloid fibrils is crucial. In this research progress report, we summarized our recent progress in the mechanism, diagnosis and treatment of $\mathrm{AD}$, including $\mathrm{A} \beta$ metal/protein/inorganic drug agent interaction mechanism, targeting inhibitors, multifunctional therapeutic agents, and their biological effects. It is expected to explore a new way for the prevention and treatment of $\mathrm{AD}$ and construction of a more efficient treatment system.
\end{abstract}

Keywords: Alzheimer's disease, amyloid $\beta$-peptides, aggregation, intermolecular interaction mechanism, multifunctional therapeutic agents

doi: $10.1360 / \mathrm{N} 032018-00081$ 